\title{
c $\therefore, \cdots$ 02068 \\ HELIOSEISMIC CONSTRAINTS ON THE GRADIENT OF ANGULAR VELOCITY AT THE BASE OF THE SOLAR CONVECTION ZONE
}

\author{
W. W. Hansen Fxperimental Physies Laboraty, A. G. Kosovichev \\ Received 1996 May 20; accepted 1996 July 3 . \\ Received 1996 May 20; accepted 1996 July 3
Rater
}

\begin{abstract}
The layer of transition from the nearly rigid rotation of the radiative interior to the latitudinal differential splitting coefficients of the $p$-mode frequencies, obtained the internal dynamics of the Sun. Using rotational we have found that the thickness of the transitional layer is during 1986-1990 at the Big Bear Solar Observatory, of the transition occurs beneath the adiabatically stratified $0.09 \pm 0.04$ solar radii $(63 \pm 28 \mathrm{Mm})$, and that most dynamo theories of the $22 \mathrm{yr}$ solar activity cycle.
\end{abstract} Subject headings: convection - methods: data an Sun: rotation

\section{INTRODUCTION}

Helioseismology has established the existence of a layer of strong gradients of angular velocity at the base of the solar convection zone (e.g., Brown et al. 1989; Goode et al. 1991; Tomczyk, Schou, \& Thompson 1995). This layer separates the convection zone exhibiting strong latitudinal differential rotation from the radiative interior rotating almost rigidly. Turbulence generated in the layer is likely to mix material in the upper radiative zonc, resulting in the observed deficit of $\mathrm{Li}$ and Be (see, e.g., Zahn 1992). However, the theoretical estimates of the precise location and the thickness of the transition layer ("tachocline") depend on details of turbulent energy and momentum transport, and are uncertain (Spiegel \& Zahn
1992).

Perhaps of the greatest interest, the transition layer is the most likely place for the solar dynamo (see, e.g. Weiss 1994). Within this layer, the toroidal magnetic flux that appears at the surface in various forms of solar activity is generated from the radial component of the poloidal field (Branderburg 1994) The toroidal flux is believed to be mainly accumulated in a thin layer just beneath the convection zone because convection would quickly destroy the toroidal flux if the layer were widely extended into the convection zone. However, as recently argued by Rüdiger \& Brandenburg (1995), this layer cannot be very thin because the period of the solar cycle, which depends on the turbulent magnetic diffusion time through the layer. would be too short. They estimated the thickness to be at least $0.05 R \approx 35 \mathrm{Mm} \approx \frac{1}{2} H_{p}$, where $R$ is the solar radius and $H_{p}$ is the local pressure scale height.

Estimates of the thickness and precise location of the transition layer by standard helioseismic inversion techniques from rotational splitting of oscillation $p$-modes are rather uncertain. Attempts to resolve the layer under global smoothness constraints lead to either an oversmoothed angular velocity profile or to spurious oscillations around the transition layer (cf. Goode et al. 1991). Thompson (1990) has demonftrated that even if the transition were discontinuous the rermal helioseismic inversions still produce a broad smooth region. To overcome these difficulties, Goode et al. (1991) assumed that the transition layer is, in fact, discontinuous and found that their model fits the helioseismic data best when the discontinuity coincides with the base of the convection zone. In this Letter, we demonstrate that the discontinuous model of solar rotation is not the best fit to the data and that a model with a transitional layer of finite thickness $(\approx 0.09 \pm 0.04 R)$ fits the data more accurately than the discontinuous model. The midpoint of this layer is found at $0.692 \pm 0.005 R$, slightly
below the convection zone.

\section{FREQUENCY SPLITTING AND SOLAR ROTATION LAW}

Observed $p$-mode rotational frequency splitting is traditionally represented in the form (Duvall, Harvey, \& Pomerantz
1986)

$$
\Delta \nu_{n, t, m}=L \sum_{k=0.1,2 \ldots} a_{2 k+1}(n, l) P_{2 k+1}\left(\begin{array}{c}
m \\
L
\end{array}\right),
$$

where $n, l$, and $m$ are the radial order, the angular degree, and the angular order of a normal mode, respectively; $L=[l(l+1)]^{1: 2}$; and $P_{k}$ are Legendre polynomials. Thus, the observational data are sets of the "odd $a$-coefficients," $a_{2 h+1}$, for mode multiplets $(n, l)$. For the modes of intermediate and high degree, $l$, which probe the convection zone, the $a$-coefficients are directly related to the radial function $A_{k}(r)$ of the solar rotation law, represented in terms of associated Legendre functions of order $1, P_{k}^{\prime}(\theta)$ (Kosovichev 1988), and expressed as

$$
\Omega(r, \theta) / 2 \pi=\sum_{k-1.1 .2 \ldots} \alpha_{k} A_{2 k+1}(r) \frac{P_{2 k+1}^{1}(\theta)}{\sin \theta},
$$
where $\alpha_{k}=(-1)^{k} k ! 2^{k} /(2 k+1) ! !$. The relation between
$a_{k}(n, l)$ and $A_{k}(r)$ is

$$
\begin{aligned}
& a_{k}(n, l)=\frac{1}{l_{n, l}} \int_{0}^{R} A_{k}(r) \\
& \quad \times\left[U_{n, l}^{2}+l(l+1) V_{n, l}^{2}-2 U_{n . l} V_{n, l}-V_{n, l}^{2}\right] \rho r^{2} d r,
\end{aligned}
$$

where $U_{n, l}$ and $V_{n, l}$ are the radial and horizontal displacement eigenfunctions of oscillation modes, $\rho(r)$ is the density, and $I_{n, l}=\int_{0}^{R}\left[U_{n, l}^{2}+l(l+1) V_{n, l}^{2}\right] \rho r^{2} d r$ is the mode inertia. The 


\section{L62}

\section{KOSOVICHEV}

contribution of the last two terms in the brackets of equation (3), resulting from the Coriolis effect, does not exceed a few percent and therefore can be treated as a small perturbation. Then we introduce coefficients $a^{\prime}$ as

$$
a_{k}(n, l) \equiv a_{k}^{\prime}(n, l)\left(1-C_{n, l}\right),
$$

where

$a_{k}^{\prime}(n, l) \approx \frac{1}{I_{n, l}} \int_{0}^{R} A_{k}(r)\left[U_{n, l}^{2}+l(l+1) V_{n, l}^{2}\right] \rho r^{2} d r$.

New coefficients $a_{k}^{\prime}(n, l)$ represent the "seismic averages" of the radial function $A_{k}$, with the weighting function proportional to the mode energy density, and $C_{n, l}$ is a constant that describes the effect of the Coriolis force (Ledoux 1951).

For high-frequency $p$-modes, $a_{k}^{\prime}(n, l)$ are essentially functions of only one asymptotic variable: the angular phase speed $\omega_{n, l} / L$ or, equivalently, the radius of the lower turning point $r_{t}$ of the modes (Gough 1984). This asymptotic behavior is demonstrated in Figure 1, in which filled circles show the seismic averages for a model of solar rotation depicted in Figure 2 . We use this asymptotic property of the splitting coefficient for the analyses of the observational data by averaging $a_{k}^{\prime}(n, l)$ over short intervals of $r_{t}$.

\section{ESTIMATES OF WIDTH AND LOCATION OF THE TRANSITION LAYER}

We have used the splitting $a$-coefficients $\left(a_{1}, a_{3}\right.$, and $\left.a_{5}\right)$ of approximately 800 multiplets of $l=5-60$ obtained by Woodard \& Libbrecht (1993) from 1986 and 1988-1990 observations made at the Big Bear Solar Observatory (BBSO). The $a$-coefficients corrected for the Coriolis effect, $a_{k}^{\prime}(n, l)$, for each year have been grouped and weighted-average computed in 16 equal intervals of $r_{t}$, between $0.5 R$ and $0.9 R$, each being $0.025 R$ wide. Then the weighted averages for the $4 \mathrm{yr}$ have been computed. The final averages, $\left\langle a_{k, j}^{\prime}\right\rangle_{\text {ots }}(j=1, \ldots, 16)$, are shown by the diamonds with the error bars in Figure 1. The variation at the base of the convection zone is particularly strong for $a_{3}^{\prime}$. Therefore, we have used this coefficient to estimate the thickness and the location of the transition layer. The absence at the base of the convection zone of a sharp variation of $a_{1}^{\prime}$, which represents the seismic average of the net angular momentum of a spherical shell, means essentially zero net torque between the radiative and convection zones (Gough 1985; Gilman, Morrow, \& DeLuca 1989). We shall discuss the limits of the variation of $a_{1}^{\prime}$ in a future publication.

Helioseismic inversions have revealed that the radiative interior essentially rotates rigidly. Therefore, in rotation law (2), functions $A_{k}(r)$ for $k>1$ are approximately equal to zero in the radiative zone. Also, the averaged $a_{3}^{\prime}(n, l)$ coefficients shown in Figure 1 suggest that $A_{3}$ is almost constant in the convection zone. Therefore, we have considered $A_{3}$ in the parametric form,

$$
A_{3}(r)=A_{3,0} \Phi(r),
$$

where $\Phi(r)=0.5\left(1+\operatorname{erf}\left[2\left(r-r_{0}\right) / w\right]\right)$, erf is the error function, $r_{0}$ is the radius of the central point of the transition zone, and $w$ is the characteristic thickness corresponding to the variation of $\Phi(r)$ from 0.08 to 0.92 . The value of $A_{3,0}$

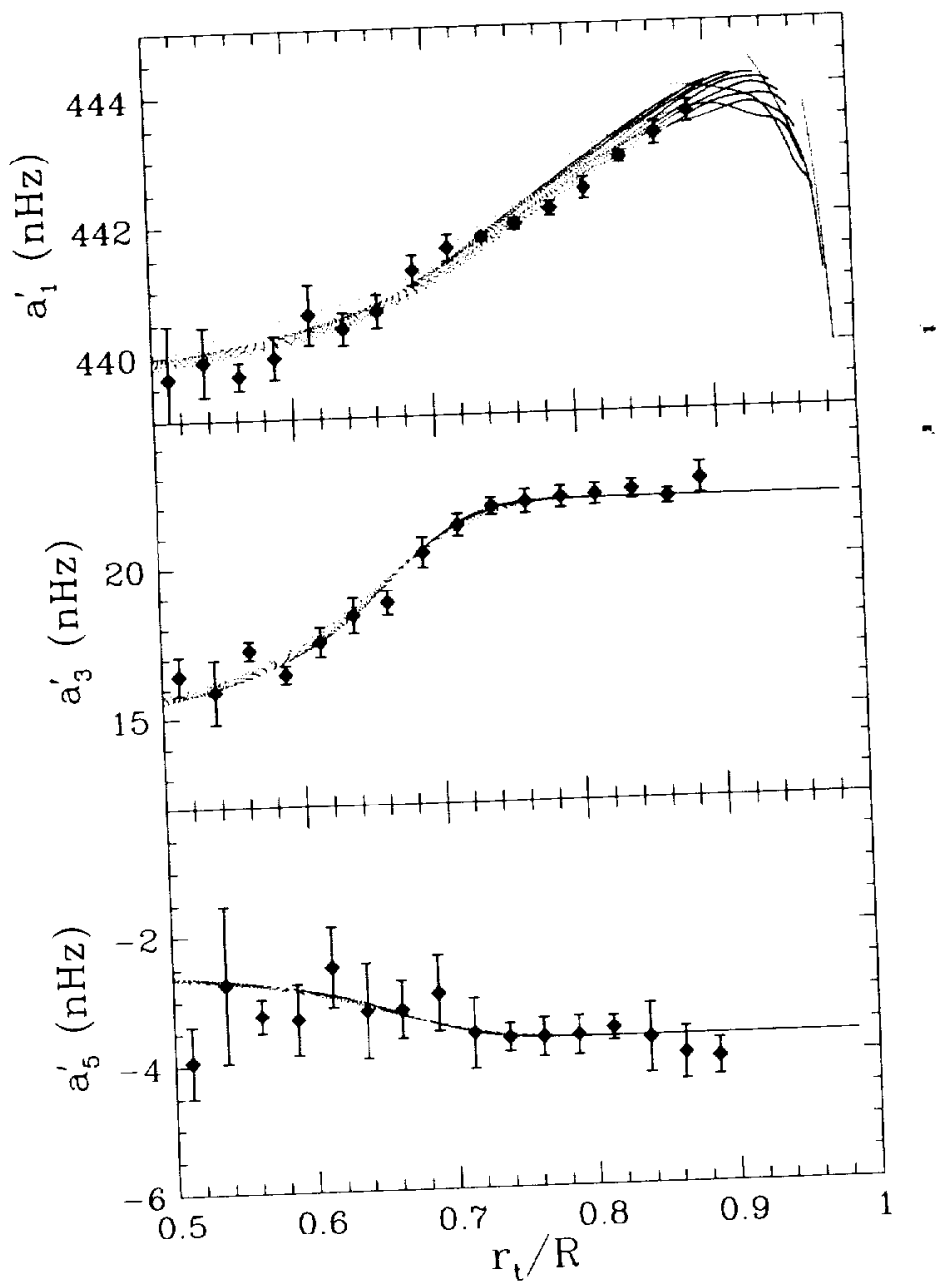

FIG. 1.-Seismic averages (eq. [5]) of the radial function, $A_{k}(r)$, of the Fic. rotation law (eq. [2]), as functions of the radius of the mode for approxipoint, $r_{f}$. Filled circles represent the theoretical values computed for aspown in mately 3000 modes of $l=5-250$ using the model of solar rotation, as shown

Fig. 2. Diamonds with the error bars show

$(\approx 22 \mathrm{nHz})$ is determined from the flat part of $a_{3}^{\prime}$ in the convection zone. Then, we computed $\left\langle a_{3}^{\prime}(n, l)\right\rangle_{\text {mod }}$ coefficients for equation (6) using equation (5) and the same averaging procedure that was applied to the BBSO data, and, finally, we determined the mean square difference,

$$
\chi^{2}\left(w, r_{0}\right)=\sum_{j=1}^{16} \frac{1}{\sigma_{j}^{2}}\left[\left\langle a_{3, j}^{\prime}\right\rangle_{\text {obs }}-\left\langle a_{3, j}^{\prime}\right\rangle_{\bmod }\right]^{2} .
$$

The function $\chi^{2}\left(w, r_{0}\right)$, shown in Figure 3, reaches a minimum of 16.8 at $r_{0} \approx 0.692 R$ and $w \approx 0.09 R$. The boundaries of the shaded area correspond to the increase of $\chi^{2}$ by 1 , or to $1 \sigma$ in $r_{0}$ and $w$. A similar result was obtained using different approximations for the transition function, $\Phi(r)$, and by choosing different intervals of $r_{t}$ for averaging the data. The analysis repeated with an extended BBSO data set $(l=5-140)$ provided to us by $\mathrm{P}$. R. Goode gave the same result.

We note that the position of the center of the transition layer is determined more accurately from the data than is the thickness. It is evident that the center of the layer is beneath 


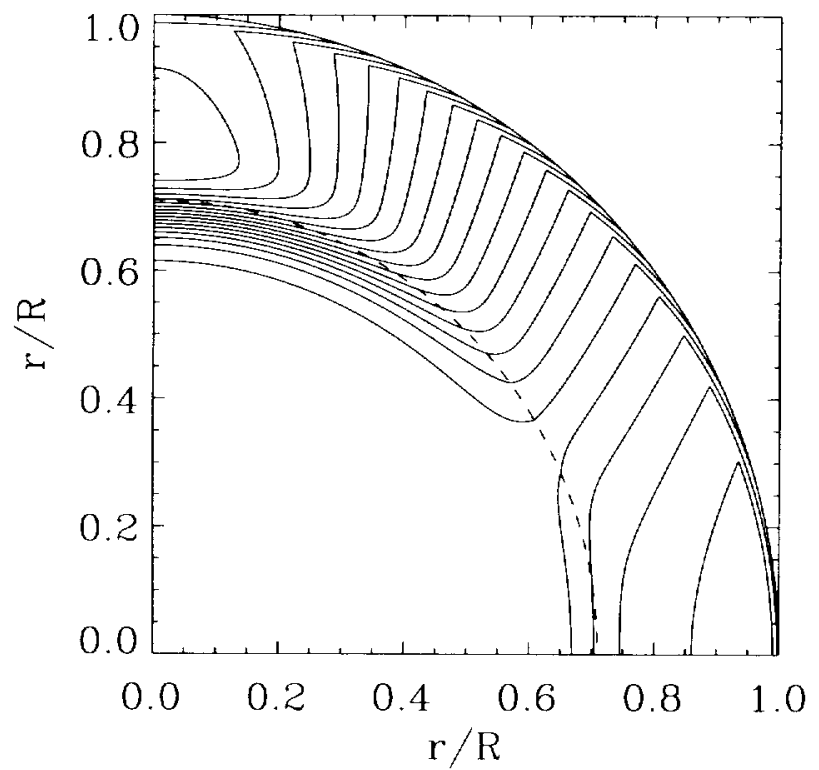

Fici. 2. Contours of $\Omega(r, \theta) / 2 \pi$ from 328 to $461 \mathrm{nHz}$, separated by $7 \mathrm{nHz}$ of a solar rotation model used in Fig. 1. Dashed curve shows the lower boundary of the convection zone. In this model, based on the first three terms of eq. (2), $A_{1}(r)$ is approximated below the convection zone by a constant and it is approximated in the convection zone by two linear functions, the first of which was chosen to fit gradually increasing $a_{1}^{\prime}$, while the second function (describing the subsurface shear layer) was found by matching the internal rotation with the surface rotation (Snodgrass 1992)-assuming the conservation of the angular momentum in the subsurface layer (Foukal \& Jokipii 1975). Estimated thickness of the subsurface layer is approximately $12 \mathrm{Mm}(0.017 R)$. Functions $A_{3}(r)$ and $A_{5}(r)$ are taken in the form of eq. (6).

the boundary of adiabatic stratification of the convection zone, which is at $0.713 \pm 0.003 R$, and coincides with the zone of the sharp variation of the sound speed (Fig. 4). For Goode \& Dziembowski's (1991) model with a discontinuity at the base of the convection zone $\left(w=0, r_{11}=0.71 R\right), x^{2} \approx 36$ is significantly higher than the minimal value. Therefore, their

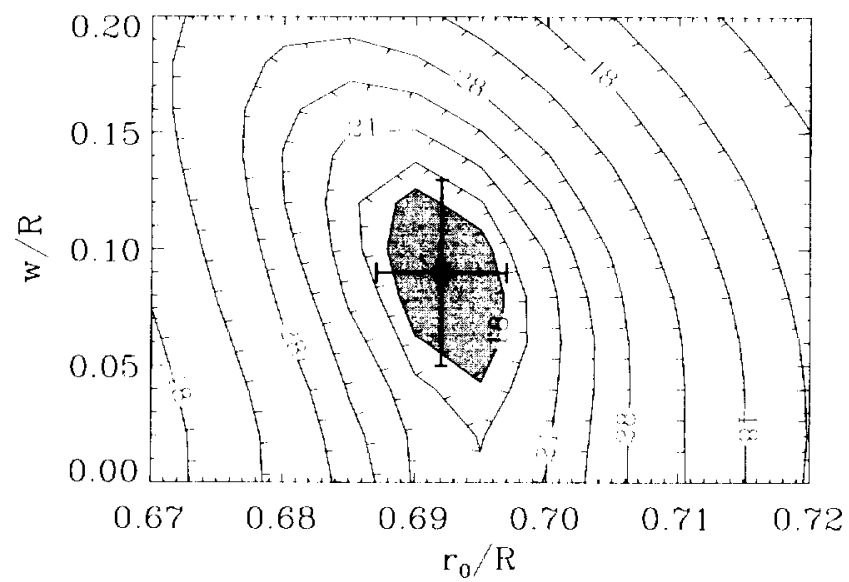

Fici. 3. Contours of $\chi^{2}\left(w, r_{11}\right)$ evaluated from eq. (7) at $\chi^{2}=18,19,21,24$, $28,36,48,64$, and $96 ; w$ is the thickness of the transition layer, $r_{i}$ is its central radius. Shaded area corresponds to the increase of $\chi^{2}$ by 1 from its minimum value, or $1 \sigma$ uncertainty in the parameters. Error bars show the I o uncertainty estimated from statistical modeling by adding Gaussian noise to the data.

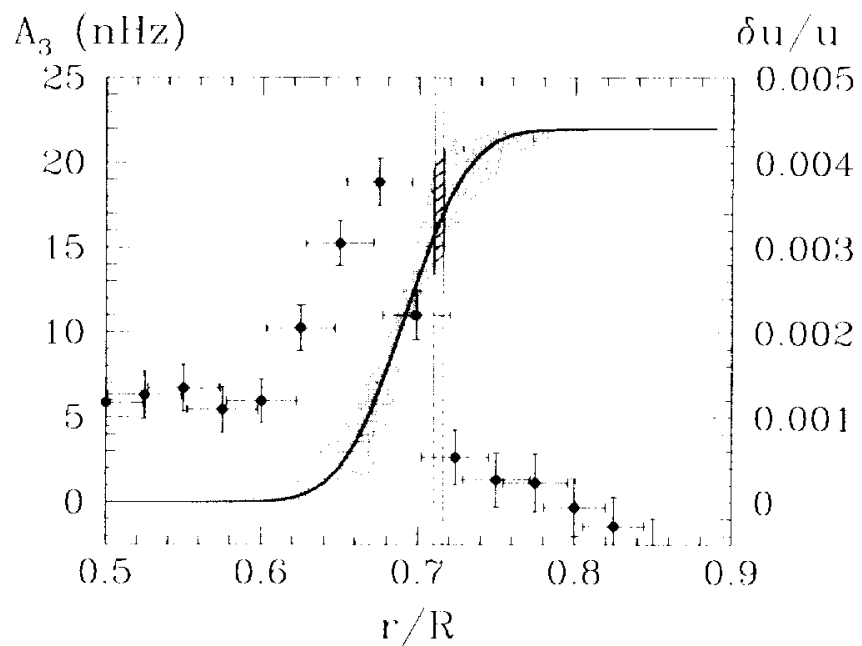

FIG. 4.-Solid curve with the shadow (indicating $1 \sigma$ uncertainty) shows the parameter $A_{3}$ of rotation law (2), estimated from the BBSO data. Points with error bars represent the variations of the ratio of the pressure to the density, $u \equiv p / p$, relative to a standard solar model, inferred from the GONG data (Gough et al. 1996). Vertical hatched column shows the location of the hase of the adiabatically stratified part of the convection zone, as determined by Christensen-Dalsgaard, Gough, \& Thompson (1991) and Kosovichev \& Fedorova (1991).

model with a very thin transition layer at the base of the convection zone can be excluded.

\section{CONCLUSION}

Estimated from the 1986-1990 BBSO solar oscillation data, the thickness $(0.09 \pm 0.04 R)$ and the central position $(0.692 \pm 0.005 R)$ of the transition layer from the nearly uniform rotation in the radiative interior to the differential rotation in the convection zone at the base of the solar convection zone are generally consistent with the requirements of the $\alpha \omega$-dynamo theory of the $22 \mathrm{yr}$ solar activity cycle (Rüdiger \& Brandenburg 1995). Our results show that most of the transition occurs beneath the zone of adiabatic convection. However, the upper boundary of the transition layer extends into the convection zone. To determine whether the transition layer coincides with the zone of convective overshoot is an important problem of helioseismology.

Another steep angular velocity gradient is likely to occur just beneath the solar surface because the angular velocity in the convection zone inferred form helioseismology is substantially higher than the angular velocity observed at the solar surface. Resolving the structure of the subsurface gradient layer is another challenging task for the new helioseismic projects, GONG and $\mathrm{SOHO}$, and would require accurate observations of rotational splitting of high-degree $p$-mode frequencies.

I thank Tom Duvall, Phil Goode, Douglas Gough, Phil Scherrer, Jesper Schou, Karel Schrijver, Alan Title, and Cornelius Zwaan for their interest and useful discussions. This research is supported by the SOI-MDI NASA contract NAG53077 at Stanford University. 


\section{REFERENCES}

Brandenburg, A. 1994, in Lectures on Solar and Planetary Dynamo, ed. M. R. E. Proctor \& A. D. Gilbert (Cambridge: Cambridge Univ. Press), 177 Brown, T. M., Christensen-Dalsgaard, J., Dziembowski, W. A., Goode, P. R., Gough, D. O., \& Morrow, C. A. 1989, ApJ, 343, 526

Christensen-Dalsgaard, J., Gough, D. O., \& Thompson, M. J. 1991, ApJ, 378, 413

Duvall, T. L., Harvey, J. W., \& Pomerantz, M. A. 1986, Nature, 321, 500

Foukal, P., \& Jokipii, J. P. 1975, ApJ, 199, L71

Gilman, P. A., Morrow, C. A., \& Deluca, E. E. 1989, ApJ, 338, 528

Goode, P. R., \& Dziembowski, W. A. 1991, Nature, 349, 223

Goode, P. R., Dziembowski, W. A., Korzennik, S. G., \& Rhodes, E. J. 1991, ApJ, 367,649

Gough, D. O. 1984, Philos. Trans. R. Soc. London, A, 313, 27

. 1985, in ESA SP-235, ESTEC, Future Missions in Solar, Heliospheric, and Space Plasma Physics, ed. E. Rolfe \& B. Battrick (Noordwijk: ESA), 183
Gough, D. O., Kosovichev, A. G., \& the GONG Team. 1996, Science, 272, 1296

Kosovichev, A. G. 1988, Bull. Crimean Astrophys. Obs., 80, 167

Kosovichev, A G. \& Fedorova, A. V. 1990, AZh, 68, 1051

Ledoux, P. 1951, ApJ, 114, 373

Rüdiger, G., \& Brandenburg, A. 1995, A\&A, 296, 557

Snodgrass, H. B. 1992, in ASP Conf. Ser. 27, The Solar Cycle, ed. K. L. Harvey (San Francisco: ASP), 205

Spiegel, E. A \& Zahn, J.-P. 1992, A\&A, 265, 106

Thompson, M. J. 1990, Solar Phys., 125, 1

Tomczyk, S., Schou, J., \& Thompson, M. J. 1995, ApJ, 448, L57

Weiss, N. O. 1994, in Lectures on Solar and Planetary Dynamo, ed. M. R. E. Proctor \& A D. Gilbert (Cambridge: Cambridge Univ. Press), 59

Woodard, M. F., \& Libbrecht, K. G. 1993, Science, 260, 1778

Zahn, J.-P. 1992, A\&A, 265, 115 